safety of the method. Complications included skin breakdown in one, red blood cells in the cerebrospinal fluid in one, hyponatremia in eight, and hypoproteinemia in two. The authors suggest that shunting should be performed if resolution of the posthemorrhagic hydrocephalus has not occurred in an infant weighing 2000 grams and if spinal fluid protein is low. (Leonhardt $A$ et al. Management of posthaemorrhagic hydrocephalus with a subcutaneous ventricular catheter reservoir in premature infants. Arch Dis Child Jan 1989; 64:24-28).

COMMENT. C. Bannister, Consultant Paediatric Neurosurgeon, Manchester, England, comments that the reservoir allows easy repeated removal of cerebrospinal fluid from the lateral ventricles and is a convenient monitor of intracranial pressure. The disadvantages include risk of infection, skin breakdown, intraventricular bleeding from rapid aspiration and pressure fluctuations, and local cortical damage secondary to catheter insertion.

\title{
SEQUELAE OF POSTHEMORRHAGIC HYDROCEPHALUS
}

The neurologic and developmental outcome of 33 low birth weight neonates with ventriculomegaly and in 39 with no ventriculomegaly after hemorrhage were evaluated prospectively in the Departments of Pediatrics and Neurosurgery, Wayne State University School of Medicine and Children's Hospital of Michigan, Detroit. Ventriculoperitoneal shunts were inserted in 23 of the 33 ventriculomegaly (VM) group infants at a mean age of 26 days. Shunt revisions were performed in 18 of the 23 children for obstruction (71) or infection (11). The total group of 72 children were followed to a miean age of 50 months. More children in the VM group had neurologic sequelae and microcephaly in comparison with the children in the non-VM group. Mild abnormalities included hypotonia and moderate and severe sequelae included spastic quadriplegia in 12 children and right hemiplegia in two children. Visual, language and hearing impairments were significantly increased in the VM group and included strabismus, myopia, nystagmus, and blindness. Developmental delay occurred in 19 patients in the VM group and in only eight in the non-VM group. Among the children with shunts, a higher incidence of sequelae occurred when lack of ventricular decompression was noted immediately after shunt insertion and when shunt infections occurred. The most important predictor of mental and motor outcome in the group with shunts was lack of ventricular decompression immediately after shunt insertion. The authors speculate that in some infants loss of brain tissue, cerebral atrophy or both may occur before insertion of the ventriculoperitoneal shunt even when the shunt is inserted early. (Shankaran S et al. Outcome after posthemorrhagic ventriculomegaly in comparison with mild hemorrhage without ventriculomegaly. JPediatr Jan 1989; 114:109-114).

COMMENT. There was no delay in the initial ventriculoperitoneal shunt insertion in this group so shunt infection and obstruction appeared to be responsible for the poor neurodevelopmental outcome. 